\title{
Tuning of Physiological Controller Motifs
}

\author{
Kristian Thorsen ${ }^{1} \quad$ Geir B. Risvoll ${ }^{1} \quad$ Daniel M. Tveit ${ }^{1} \quad$ Peter Ruoff $^{2} \quad$ Tormod Drengstig $^{1}$ \\ ${ }^{1}$ Department of Electrical Engineering and Computer Science, University of Stavanger, Norway, \\ \{kristian.thorsen, geir.risvoll, daniel.m.tveit, tormod.drengstig\}@uis.no \\ ${ }^{2}$ Center for Organelle Research, University of Stavanger, Norway, peter.ruoff@uis.no
}

\begin{abstract}
Genetic manipulation is increasingly used to fine tune organisms like bacteria and yeast for production of chemical compounds such as biofuels and pharmaceuticals. The process of creating the optimal organism is difficult as manipulation may destroy adaptation and compensation mechanisms that have been tuned by evolution to keep the organisms fit. The continued progress in synthetic biology depends on our ability to understand, manipulate, and tune these mechanisms. Concepts from control theory and control engineering are very applicable to these challenges. From a control theoretic viewpoint, disturbances rejection and set point tracking describe how adaptation mechanisms relate to perturbations and to signaling events. In this paper we investigate a set regulatory mechanisms in the form of biochemical reaction schemes, socalled controller motifs. We show how parameters related to the molecular and kinetic mechanisms influence on the dynamical behavior of disturbance rejection and set point tracking of each controller motif. This gives insight into how a molecular controller motif can be tuned to a specified regulatory response.
\end{abstract}

Keywords: bioengineering, biological systems, adaptation

\section{Introduction}

\subsection{Homeostasis, Disturbance Rejection and Set Point Tracking}

Homeostasis is described as the mechanism behind the observed adaptation of an organism in a changing environment (Cannon, 1929; Langley, 1973). From a control theoretic point of view homeostasis can be described by the properties of disturbance rejection and set point tracking.

A physiological example of disturbance rejection is the intravenous/oral glucose tolerance test (IVGTT/OGTT), where the blood glucose concentration is measured at regular intervals after injecting/eating large amounts of glucose (Ackerman et al., 1964). If the blood glucose level is above a predefined level after a certain amount of time, the patient is often diagnosed as diabetic (Ame, 2014). Over the last half century, such disturbance rejection studies are reported in a vast number of publications, see e.g. (Larsen et al., 2003; Steele, 1959), and also a large number of mathematical models are made with the aim to capture the glucose and insulin dynamics, see e.g. the comprehensive review of (Ajmera et al., 2013). Both OGTT and IVGTT represent an impulse (or short time pulse) disturbance perturbation, whereas the chronic infusion of glucose (Topp et al., 2004) represent a stepwise disturbance. Another physiological example of adaptation to a stepwise perturbation change is the adaptation of light sensitivity of the eye, which includes both a compensatory change in pupillary size and an adaptation of the photochemical system in the rods and cones (Guyton and Hall, 2006).

Physiological examples where set point tracking is investigated are relatively rare, although set point determining mechanisms with respect to body temperature and metabolism have beed discussed (Briese, 1998; St Clair Gibson et al., 2005).

Regulatory mechanisms can today be synthetically modified or added to make organism better suitable for a specific job. Still, engineering of biochemical networks has not yet achieved the status and robustness as engineering of electrical and mechanical systems (Ang et al., 2010). From a synthetic biology perspective (Ang and McMillen, 2013; Ang et al., 2013), it is thus of vital importance to have insight into the biochemical mechanisms behind physiological regulatory systems. One possible way to gain such insight is to analyze both the disturbance rejection and set point tracking dynamics of such systems in vivo, as well as doing in silico studies based on different model candidates. The latter approach is a well known technique used in control engineering. We will in this paper start with the simplest form of biochemical networks with regulatory function and identify by model analysis and simulation how the dynamic response of such networks can be tuned.

\subsection{Controller Motifs}

A biochemical network with regulatory properties must in its simplest form include at least two components, i.e., state variables, one controlled component and a controller component. The controller component acts on the controlled component in a way that compensates for external disturbances. We have earlier presented a collection of simple two-component regulatory networks (Drengstig et al., 2012; Thorsen et al., 2013), and we have used the name controller motifs to describe them. These motifs consist of two chemical species, $A$ and $E$, 
both of them being formed and turned over. A may represent an intracellular compound which is subject to disturbances in the form of e.g. uncontrolled diffusive transport of $A$ in and out of the cell, and $E$ may represent a membrane bound compound such as a transporter protein as shown in Figure 1. Like many cellular compounds which is subject to strict regulation (due to e.g. toxicity if present in large amount), the concentration of $A$ should not exceed or be less than some limits. By connecting the compounds $A$ and $E$ through cellular signaling events such as activation and inhibition, species $A$ becomes the controlled variable, while species $E$ becomes the manipulated variable.

Based on the direction of the $E$-mediated flow, the motifs fall into two categories termed inflow and outflow controllers. The complete set of possible inflow and outflow controller motifs are shown in Figure 2, and the steady state properties of these controllers were presented in (Drengstig et al., 2012). Based on the type of $E$-mediated inflow or outflow, the controllers are further divided into activating (inflow $1 / 3$ and outflow 5/7) or inhibiting (inflow 2/4 and outflow 6/8) controller type, indicated by grey and white background in Figure 2, respectively.

In the following we will show how the parameters of the controller motifs, i.e. rate constants, Michaelis-Menten constants, activation constants and inhibition constants, influence on the dynamic performance, and show how it is possible to adjust the system's response similar to the tuning of industrial control systems.

\section{Results}

\subsection{Dynamic Properties of Controller Motifs}

The dynamic properties of a two component biochemical system (second order system) can be described in terms of the undamped natural frequency $\omega_{n}$ and the damping ratio $\zeta$. To illustrate how these two parameters relate to the regulatory mechanisms in Figure 2, we use outflow controller 5 as an example. For unique identification, we
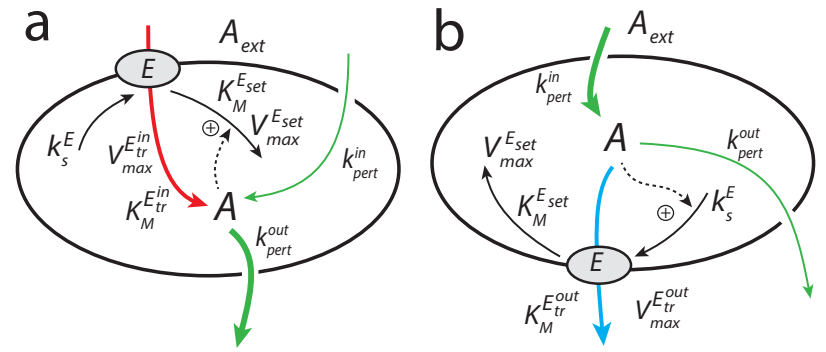

Figure 1. Illustration of a cell with a compound $A$ being under homeostatic control by an inflow controller (panel a) or an outflow controller (panel b). Panel a: An inflow controller compensate for outflow perturbations, $k_{\text {pert }}^{\text {out }}$ (thick green line), in $A$ by adding more $A$ through an $E$-mediated inflow (red line). Panel b: An outflow controller compensate for inflow perturbations, $k_{\text {pert }}^{\text {in }}$ (thick green line), in $A$ by removing excess of $A$ through an $E$-mediated outflow (blue line).

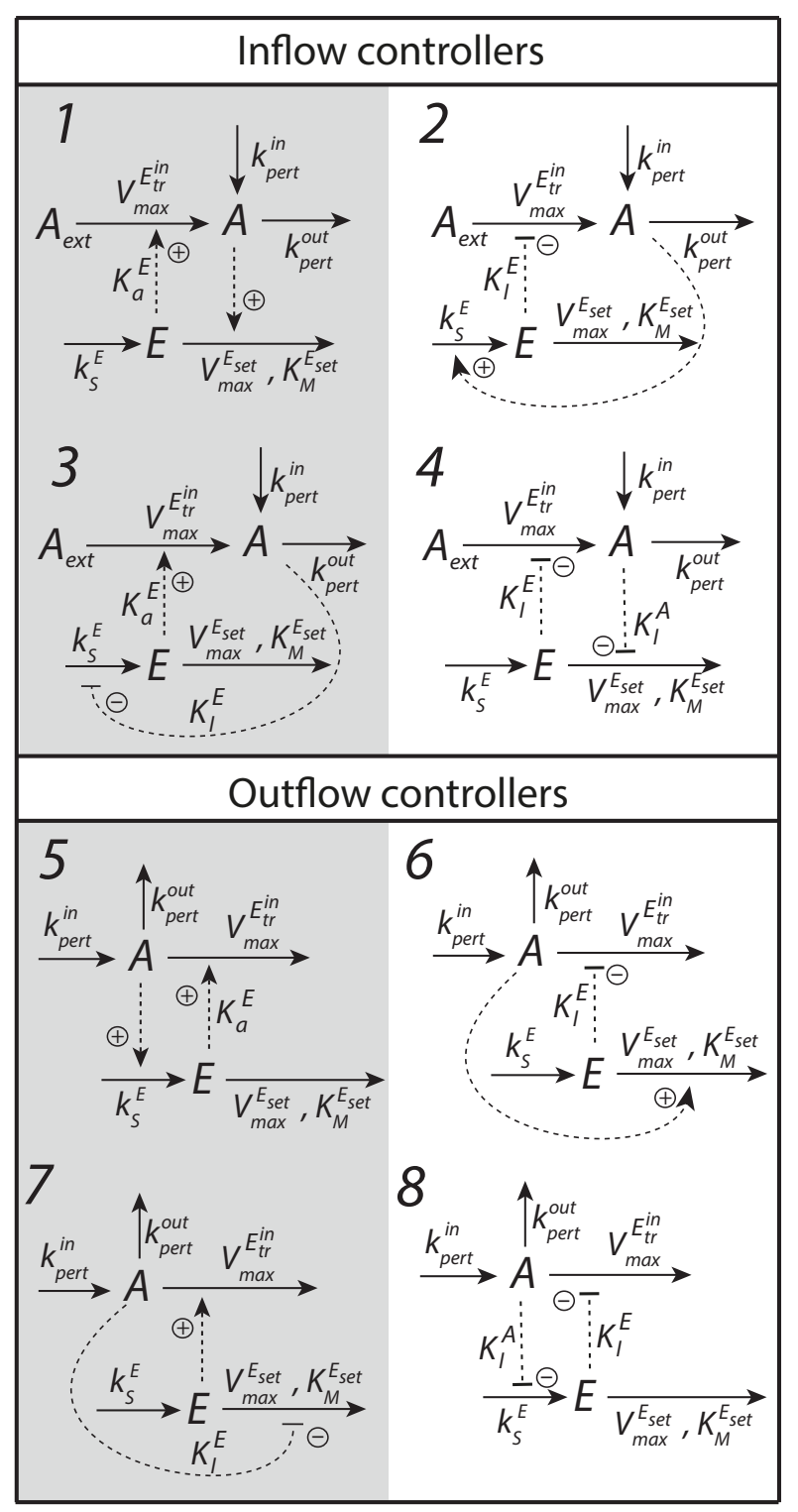

Figure 2. Set of two-component homeostatic controller motifs (Drengstig et al., 2012) classified as inflow and outflow controllers, where grey or white background indicate activating or inhibiting controller types, respectively.

apply subscript ${ }_{5}$ on the appropriate parameters and variables, and hence, the nonlinear rate equations for an outflow controller 5 are given as (Drengstig et al., 2012):

$$
\begin{gathered}
\dot{A}=k_{\text {pert }}^{\text {in }}-k_{\text {pert }}^{\text {out }} \cdot A-V_{\text {max }}^{E_{t r, 5}} \cdot A \cdot \frac{E_{5}}{\left(K_{a}^{E_{5}}+E_{5}\right)} \\
\dot{E}_{5}=k_{s}^{E_{5}} \cdot A-\frac{V_{\text {max }, 5}^{E_{\text {set }}} \cdot E_{5}}{\left(K_{M}^{E_{\text {set }, 5}}+E_{5}\right)}
\end{gathered}
$$

As discussed in (Drengstig et al., 2012), the set point $A_{\text {set }}^{\text {out }, 5}$ is found by assuming ideal (theoretical) conditions, i.e. $K_{M}^{E_{s e t, 5}}=0$ in (2), to give $A_{\text {set }}^{\text {out }, 5}=\frac{V_{\text {max }, 5}^{E_{\text {mex }}}}{k_{s}^{E_{5}}}$. Once the theoretical set point is established, we re-assume realistic conditions and reorganize (2) into the integral control law 
equation $\dot{E}_{5}=G_{i, 5} \cdot\left(A_{\text {set }}^{\text {out }, 5}-A_{\text {meas }}\right)$. This allows us to identify the integral controller gain $G_{i, 5}$ and the measurement signal $A_{\text {meas }}$ as:

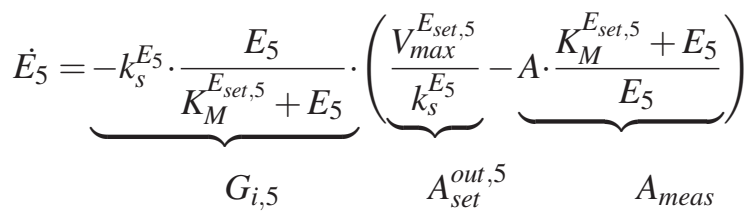

Note that the measurement signal $A_{\text {meas }}$ actually includes information about the control signal $E_{5}$ which is not common in industrial control engineering. Note also that as long as $K_{M}^{E_{s e t, 5}}>0$, the actual value of $A$ will be less than the theoretical set point $A_{\text {set }}^{\text {out }, 5}$. Nevertheless, the set point tracking properties are good since the control error $e$, calculated as:

$$
e=\left(A_{\text {set }}^{\text {out }, 5}-A_{\text {meas }}\right)
$$

is zero. The difference between the actual level of $A$ and the theoretical set point $A_{\text {set }}$ is termed inaccuracy (Thorsen, 2015). A general result valid for all controller motifs is that both rate constants for synthesis and degradation of $E$, i.e. $k_{s}^{E}$ and $V_{\text {max }}^{E_{\text {set }}}$, are a part of the set point $A_{\text {set }}$ (Drengstig et al., 2012). At the same time, one of these rate constants is also a part of the integral controller gain $G_{i}$.

In order to identify the parameters $\omega_{n, 5}$ and $\zeta_{5}$, we once again assume ideal conditions, i.e. $K_{M}^{E_{s e t, 5}}=0$, and continue by linearizing the model in (1) and (2) around an arbitrary working point $A_{s s}$ and $E_{5, s s}$. Since the set point consist of two individual parameters, i.e. $k_{s}^{E}$ and $V_{\text {max }}^{E_{\text {sex }}}$, we select $V_{m a x}^{E_{\text {set }}}$ to be our input. We then find the closed looped transfer function from the Laplace transformed input $\Delta V_{\max }^{E_{\text {set }, 5}}(s)$ to the Laplace transformed output $\Delta A(s)$ as:

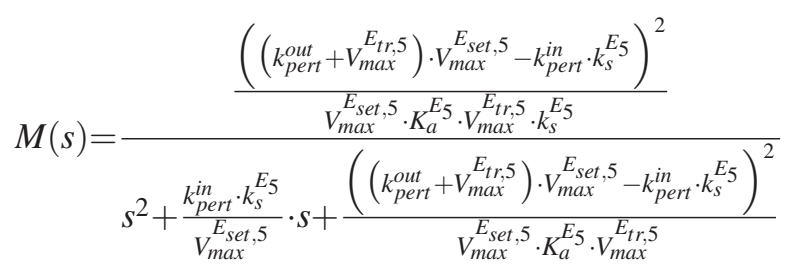

Using that $V_{\text {max }}^{E_{\text {set }, 5}}=k_{s}^{E_{5}} \cdot A_{\text {set }}^{\text {out }, 5}$, we find $\omega_{n, 5}$ and $\zeta_{5}$ as:

$$
\begin{gathered}
\omega_{n, 5}=\frac{\sqrt{k_{s}^{E_{5}}} \cdot\left(\left(k_{\text {pert }}^{\text {out }}+V_{\text {max }}^{E_{t r, 5}}\right) \cdot A_{\text {set }}^{\text {out }, 5}-k_{\text {pert }}^{\text {in }}\right)}{\sqrt{K_{a}^{E_{5}} \cdot V_{\text {max }}^{E_{t r, 5}} \cdot A_{\text {set }}^{\text {out }, 5}}} \\
\left.\zeta_{5}=\frac{k_{\text {pert }}^{\text {in }} \sqrt{K_{a}^{E_{5}} \cdot V_{\text {max }}^{E_{t r, 5}}}}{2 \cdot \sqrt{V_{\text {max }}^{E_{\text {set }, 5}}} \cdot\left(\left(k_{\text {pert }}^{\text {out }}+V_{\text {max }}^{E_{t r, 5}}\right) \cdot A_{\text {set }}^{\text {out }, 5}-k_{\text {pert }}^{\text {in }}\right)}\right)
\end{gathered}
$$

From (4) and (5) we see that, depending on the perturbation levels (inflow versus outflow perturbations), it is possible to obtain negative values for $\omega_{n, 5}$ and $\zeta_{5}$.
These negative values correspond to circumstances where the perturbation levels are such that the controller breaks down (Drengstig et al., 2012). Breakdown occurs when the net inflow perturbation is larger than the capacity of the outflow controller, i.e., greater than the maximum of the compensatory flow. In this case there is no stable equilibrium in the system and $A$ integrates towards infinity. Such a state is unwanted and may very likely be toxic for the cell. In this case the values of $\omega_{n, 5}$ and $\zeta_{5}$ are invalid and have no physical meaning. Table 1 gives a summary of $\omega_{n}$ and $\zeta$ for the four inflow and four outflow controllers, together with the expression for each set point $A_{\text {set }}$.

Note that there is a close relationship between the expressions for $\zeta$ and $\omega_{n}$ for each controller, and thus, it is not possible to specify both $\zeta$ and $\omega_{n}$ independently.

Since controller 5 is an outflow controller, the inflow perturbation $\Delta k_{\text {pert }}^{\text {in }}(s)$ is considered the main disturbance, and the transfer function characterizing the disturbance rejection properties is:

$$
N(s)=\frac{s}{s^{2}+\frac{k_{p e r t}^{\text {in }} \cdot k_{s}^{E_{5}}}{V_{\text {max }}^{E_{\text {set }}}} \cdot s+\frac{\left(\left(k_{\text {pert }}^{\text {out }}+V_{\max }^{E_{t r, 5}}\right) \cdot V_{\max }^{E_{\text {set }, 5}}-k_{\text {pert }}^{\text {in }} \cdot k_{s}^{E_{5}}\right)^{2}}{V_{\max }^{E_{\text {set }, 5}} \cdot K_{a}^{E_{5}} \cdot V_{\max }^{E_{t r .5}}}}
$$

As expected, this transfer function has a zero in the origin, implicating homeostatic behavior and perfect adaptation (Drengstig et al., 2008).

\subsection{Tuning of Individual Controllers}

As shown in (Drengstig et al., 2012), the steady state performance of the individual controllers were found to be identical, given a certain set of parameter values. A related issue is to determine whether it is possible to tune the controllers to obtain identical dynamical performance using the theoretical design parameters in Table 1. Such tuning will be useful in synthetic biology. Also on a more fundamental level, if such tuning is possible it implies that it is impossible to infer the underlining network structure, i.e., the particular controller motif, responsible for an observed adaptive process by measuring the dynamical properties of the controlled variable alone.

We have selected to use the rate constants of the synthesis and degradation of the controller species, $k_{s}^{E}$ and $V_{\text {max }}^{E_{s e t}}$, together with the rate constant of the $E$-mediated compensatory flow $V_{\text {max }}^{E_{t r}}$, as our tunable parameters. These parameters are relatively easy to tune from the perspective of synthetic biology and offer a greater tunable range than the parameters associated with the nonlinearities in the model $\left(K_{a}^{E}, K_{I}^{A}\right.$, and $\left.K_{I}^{E}\right)$. To discuss one of the tunable parameters, the rate constant for synthesis of $E, k_{s}^{E}$, can in practice be modified by altering the promoter of the gene coding for $E$. One way to do this is a fixed tuning of the promoter itself, e.g. the $\mathrm{Cu}$-dependent promoter of the CUP1-gene of Saccharomyces Cerevisiae can be modified by mutations to show wide range of different induction ratios (Thiele and Hamer, 1986). Another option is to use a dual mode promoter, a type of promoter who's regulation 
Table 1. The set point $A_{\text {set }}$, natural undamped frequency $\omega_{n}$ and damping ratio $\zeta$ for controller motifs 1-8 in Figure 2 under theoretical conditions, i.e. $K_{M}^{E_{s e t}}=0$. For each controller we have added a subscript to the parameters for unique identification.

\begin{tabular}{|c|c|c|}
\hline$A_{s e t}^{i n, 1}=\frac{k_{s}^{E_{1}}}{V_{\max }^{E_{s e t}, 1}}$ & $\omega_{n, 1}=\frac{\sqrt{V_{\text {max }}^{E_{\text {set }}}}\left(k_{\text {pert }}^{\text {in }}-k_{\text {pert }}^{\text {out }} A_{\text {set }}^{\text {in, } 1}+V_{\text {max }}^{E_{t r, 1}} A_{\text {ext }}\right)}{\sqrt{K_{a}^{E_{1}} V_{\text {max }}^{E_{t r 1}} A_{\text {ext }}}}$ & $\zeta_{1}=\frac{k_{\text {pert }}^{\text {out }} \sqrt{K_{a}^{E_{1}} V_{\text {max }}^{E_{t r, 1}} A_{\text {ext }}}}{2 \sqrt{V_{\text {max }}^{E_{\text {set }}}\left(k_{\text {pert }}^{\text {in }}-k_{\text {pert }}^{\text {out }} t_{\text {set }}^{\text {in, } 1}+V_{\text {max }}^{E_{t r 1} 1} A_{\text {ext }}\right)}}$ \\
\hline$A_{s e t}^{i n, 2}=\frac{V_{m a x}^{E_{s e t}, 2}}{k_{s}^{E_{2}}}$ & $\omega_{n, 2}=\frac{\sqrt{k_{s}^{E_{2}}}\left(k_{\text {pert }}^{\text {out }} \text { set }_{\text {set }}^{\text {in }}-k_{\text {pert }}^{\text {in }}\right)}{\sqrt{K_{I}^{E_{2}} V_{\text {max }}^{E_{\text {tr.2 }}} A_{\text {ext }}}}$ & $\zeta_{2}=\frac{k_{\text {pert }}^{\text {out }} \sqrt{K_{I}^{E_{2}} V_{\text {max }}^{E_{t r}, 2} A_{\text {ext }}}}{2 \sqrt{k_{s}^{E_{2}}}\left(k_{\text {pert }}^{\text {out }} A_{\text {set }}^{\text {in, }}-k_{\text {pert }}^{\text {in }}\right)}$ \\
\hline$A_{s e t}^{i n, 3}=\frac{k_{s}^{E_{3}} K_{I}^{A}}{V_{\max }^{E_{s e t}, 3}}-K_{I}^{A}$ & $\omega_{n, 3}=\frac{\sqrt{V_{\text {max }}^{E_{\text {set }, 3}}}\left(k_{\text {pert }}^{\text {in }}-k_{\text {pert }}^{\text {out }} A_{\text {set }}^{\text {in, } 3}+V_{\text {max }}^{E_{t r .3}} A_{e x t}\right)}{\sqrt{\left(K_{I}^{A}+A_{\text {set }}^{\text {in, }}\right) V_{\text {max }}^{E_{t r .}, 3} K_{a}^{E_{3}} A_{\text {ext }}}}$ & $\zeta_{4}=\frac{k_{p e r t}^{\text {out }} \sqrt{\left(K_{I}^{A}+A_{\text {set }}^{i n, 4}\right) V_{\text {max }}^{E_{t r, 4}} K_{I}^{E_{4}} A_{\text {ext }}}}{2 \sqrt{k_{s}^{E_{4}}\left(k_{\text {pert }}^{\text {out }} A_{\text {set }}^{i n, 4}-k_{\text {pert }}^{\text {in }}\right)}}$ \\
\hline$A_{\text {set }}^{i n, 4}=\frac{V_{\max }^{E_{\text {set }, 4}} K_{I}^{A}}{k_{s}^{E_{4}}}-K_{I}^{A}$ & $\omega_{n, 4}=\frac{\sqrt{k_{s}^{E_{4}}}\left(k_{\text {pert }}^{\text {out }} A_{\text {set }}^{\text {in, }}-k_{\text {pert }}^{\text {in }}\right)}{\sqrt{\left(K_{I}^{A}+A_{\text {set }}^{\text {in, }}\right) V_{\text {max }}^{E_{t r i},} K_{I}^{E_{4}} A_{\text {ext }}}}$ & $\zeta_{3}=\frac{k_{\text {pert }}^{\text {out }} \sqrt{\left(K_{I}^{A}+A_{\text {set }}^{\text {in } 3}\right) V_{\text {max }}^{E_{t r \cdot 3} K_{a}^{E_{3}} E_{3}} A_{e x t}}}{2 \sqrt{V_{\text {max }}^{E_{\text {set }}, 3}\left(k_{\text {pert }}^{\text {in }}-k_{\text {pert }}^{\text {out }} A_{\text {set }}^{\text {in, } 3}+V_{\text {max }}^{E_{t r .3}} A_{\text {ext }}\right)}}$ \\
\hline$A_{\text {set }}^{\text {out }, 5}=\frac{V_{\text {mat }}^{E_{\text {set }}}}{k_{s}^{E_{5}}}$ & $\omega_{n, 5}=\frac{\sqrt{k_{s}^{E_{5}}}\left(\left(k_{\text {pert }}^{\text {out }}+V_{\text {max }}^{E_{t r .5}}\right) A_{\text {set }}^{\text {out }, 5}-k_{\text {pert }}^{\text {in }}\right)}{\sqrt{K_{a}^{E_{5}} V_{\text {max }}^{E_{t r .5}} A_{\text {set }}^{\text {out }, 5}}}$ & $\zeta_{5}=\frac{k_{\text {pert }}^{\text {in }} \sqrt{K_{a}^{E_{5}} V_{\text {max }}^{E_{t r .5}}}}{2 \sqrt{V_{\text {max }}^{E_{\text {set }}}\left(\left(k_{\text {pert }}^{\text {out }}+V_{\text {max }}^{E_{t r a x}}\right) A_{\text {set }}^{\text {out }, 5}-k_{\text {pert }}^{\text {in }}\right)}}$ \\
\hline$A_{\text {set }}^{\text {out }, 6}=\frac{k_{s}^{E_{6}}}{V_{\text {max }}}$ & 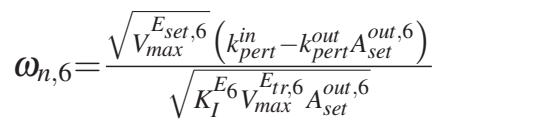 & $\zeta_{6}=\frac{k_{\text {pert }}^{\text {in }} \sqrt{K_{I}^{E_{6}} V_{\text {max }}^{E_{t r}, 6}}}{2 \sqrt{k_{s}^{E_{6}}\left(k_{\text {pert }}^{\text {in }}-k_{\text {pert }}^{\text {out }} A_{\text {set }}^{\text {out }, 6}\right)}}$ \\
\hline$A_{\text {set }}^{\text {out }, 7}=\frac{V_{\text {sex }}^{E_{\text {set }}, 7} K_{I}^{A}}{k_{s}^{E_{7}}}-K_{I}^{A}$ & $\omega_{n, 7}=\frac{\sqrt{k_{s}^{E_{7}}}\left(\left(k_{\text {pert }}^{\text {out }}+V_{\text {max }}^{E_{t r, 7}}\right) A_{\text {set }}^{\text {out }, 7}-k_{\text {pert }}^{\text {in }}\right)}{\sqrt{\left(A_{\text {set }}^{\text {out }, 7}+K_{I}^{A}\right) V_{\text {max }}^{E_{\text {tr. }}, 7} K_{a}^{E_{7}} A_{\text {set }}^{\text {out }, 7}}}$ & $\zeta_{7}=\frac{k_{\text {pert }}^{\text {in }} \sqrt{\left(A_{\text {set }}^{\text {out }, 7}+K_{I}^{A}\right) V_{\text {max }}^{E_{t r .}, K_{a}^{E_{7}}}}}{2 \sqrt{k_{s}^{E_{7}} A_{\text {set }}^{\text {out }, 7}\left(\left(k_{\text {pert }}^{\text {out }}+V_{\text {max }}^{E_{t r, 7}}\right) A_{\text {set }}^{\text {out }, 7}-k_{\text {pert }}^{\text {in }}\right)}}$ \\
\hline$A_{\text {set }}^{\text {out }, 8}=\frac{k_{s}^{E_{8}} K_{I}^{A}}{V_{\text {mat }}^{E_{\text {set }}}}-K_{I}^{A}$ & 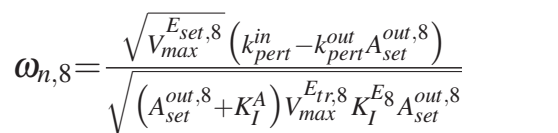 & $\zeta_{8}=\frac{k_{\text {pert }}^{\text {in }} \sqrt{\left(A_{\text {set }}^{\text {out }, 8}+K_{I}^{A}\right) V_{\text {max }}^{E_{t r}, 8} K_{I}^{E_{8}}}}{2 \sqrt{V_{\text {max }}^{E_{\text {set }}, 8} A_{\text {set }}^{\text {out }, 8}\left(k_{\text {pert }}^{\text {in }}-k_{\text {pert }}^{\text {out }} A_{\text {set }}^{\text {out }, 8}\right)}}$ \\
\hline
\end{tabular}

of protein production depends on two activators. One activator would be the control variable $A$ and another would be a chemical compound that can be meticulously added to the growth medium to achieve a certain level of gene transcription and production of $E$, represented in the model as the value of $k_{s}^{E}$. One such promoter controlled by Testosterone and IPTG (isopropyl $\beta$-D-1-thiogalactopyranoside) has recently been developed (Mazumder and McMillen, 2014).

In order to best tune the parameters we have to know about the operational limits of the system. For this purpose, we define as in (Drengstig et al., 2012) an upper limit for the maximum compensatory flux, $j_{A, \max }=10$, corresponding to a maximum level of $E_{\max }=15$ for the activating controllers $1,3,5$ and 7 , and corresponding to $E_{\text {min }}=0$ for the inhibiting controllers 2, 4, 6 and 8. We assume further that the set point of $A$ is $A_{\text {set }}=1.0$, the external concentration is $A_{\text {ext }}=2$. The kinetic constants for activation and inhibition are chosen to avoid saturation effects: $K_{a}^{E}=2, K_{I}^{A}=0.1$ and $K_{I}^{E}=1.0$. Moreover, the working point of perturbations is specified as $k_{\text {pert }}^{\text {in }}=2 / k_{\text {pert }}^{\text {out }}=5$ for inflow controllers and $k_{\text {pert }}^{\text {in }}=5 / k_{\text {pert }}^{\text {out }}=2$ for outflow controllers. Given these overall system parameters, the tuning procedure of each individual controller motif is based on specifying $\zeta$ (or $\omega_{n}$, but not both) in a similar way as the pole placement method, and determine the last three parameter values of each motif, i.e. $V_{\text {max }}^{E_{t r}}, V_{\text {max }}^{E_{\text {set }}}$ and $k_{s}^{E}$.

To illustrate, we specify two different dynamical responses in the concentration of $A$ for a step in $A_{\text {set }}$, i.e. one critically damped $(\zeta=1)$ and one underdamped $(\zeta=0.2$ corresponding to $50 \%$ overshoot) response. A strongly underdamped system overshoots when adapting a change in set point, but shows considerably better disturbance rejection than a critically damped system. Thus, tuning for the latter may be of interest in many biological systems.

We illustrate the procedure in detail by continuing on the outflow controller 5 example, and start by considering the rate expression for the compensatory flux, $j_{A}$, from (1):

$$
j_{A}=V_{\max }^{E_{t r, 5}} \cdot A \cdot \frac{E_{5}}{\left(K_{a}^{E_{5}}+E_{5}\right)}
$$

By setting $j_{A}=j_{A, \max }=10$ and inserting $E_{5}=E_{5, \max }=15$, $A=A_{\text {set }}^{\text {out }, 5}=1$ and $K_{a}^{E_{5}}=2$ into (6), gives $V_{\max }^{E_{t r, 5}}=11.33$. Using the mathematical expressions for $A_{\text {set }}^{\text {out }, 5}$ and $\zeta_{5}$ tabulated in Table 1, we find $V_{\max }^{E_{\text {set, }}}=2.04$ and $k_{s}^{E_{5}}=2.04$ for 
$\zeta_{5}=1$ and $V_{\max }^{E_{\text {set }, 5}}=51.0$ and $k_{s}^{E_{5}}=51.0$ for $\zeta_{5}=0.2$, see Table 2 .

Table 2. The parameters $V_{\text {max }}^{E_{t r}}, V_{\text {max }}^{E_{\text {set }}}, k_{s}^{E}$ and the integral controller gain $G_{i}$ (in grey) for each controller motif specified for critical damped response $\zeta=1$ and underdamped response $\zeta=0.2$. The other parameters are defined in the main text.

\begin{tabular}{|c|c|c|c|c|c|}
\hline & & $V_{\max }^{E_{t r}}$ & $V_{\max }^{E_{\text {set }}}$ & $k_{s}^{E}$ & $G_{i}$ \\
\hline \multirow{4}{*}{ 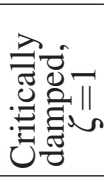 } & Inflow 1 & 5.67 & 2.04 & 2.04 & 2.04 \\
\hline & Inflow 2 & 5.00 & 6.94 & 6.94 & -6.94 \\
\hline & Inflow 3 & 5.67 & 2.24 & 24.68 & 2.04 \\
\hline & Inflow 4 & 5.00 & 84.03 & 7.64 & -6.94 \\
\hline \multirow{4}{*}{ 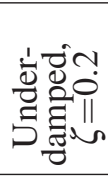 } & Inflow 1 & 5.67 & 51.0 & 51.0 & 51.0 \\
\hline & Inflow 2 & 5.00 & 173.6 & 173.6 & -173.6 \\
\hline & Inflow 3 & 5.67 & 56.1 & 617.1 & 51.0 \\
\hline & Inflow 4 & 5.00 & 2100.7 & 191.0 & -173.6 \\
\hline \multirow{4}{*}{ 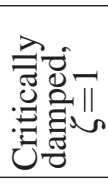 } & Outflow 5 & 11.33 & 2.04 & 2.04 & -2.04 \\
\hline & Outflow 6 & 10.00 & 6.94 & 6.94 & 6.94 \\
\hline & Outflow 7 & 11.33 & 24.68 & 2.24 & -2.04 \\
\hline & Outflow 8 & 10.00 & 7.64 & 84.03 & 6.94 \\
\hline \multirow{4}{*}{ 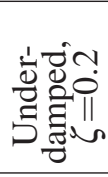 } & Outflow 5 & 11.33 & 51.0 & 51.0 & -51.0 \\
\hline & Outflow 6 & 10.00 & 173.6 & 173.6 & 173.6 \\
\hline & Outflow 7 & 11.33 & 617.1 & 56.1 & -51.0 \\
\hline & Outflow 8 & 10.00 & 191.0 & 2100.7 & 173.6 \\
\hline
\end{tabular}

This corresponds to an integral controller gain of $G_{i, 5}=-2.04$ and $G_{i, 5}=-51.0$, respectively, and a response time of $T_{r} \approx 0.8$ seconds $\left(\omega_{n, 5}=2.5\right)$ and $T_{r} \approx 0.1$ seconds $\left(\omega_{n, 5}=12.5\right)$. The simulation results shown as black curves in panels $\mathbf{c}$, and $\mathbf{d}$ in Figure 3, verify the tuning specifications, both with respect to overshoot and response time.

In order to compare the individual performance of each controller, the above described tuning specifications are applied for all controllers, and the results are shown in Table 2 and verified by simulation in Figure 3 .

Note the identical values for $G_{i}$ (greyed out in Table 2) for all the activating (inflow $1 / 3$ and outflow 5/7) and all the inhibiting (inflow 2/4 and outflow 6/8) controllers, respectively. Note also the opposite signs for activating and inhibiting inflow and outflow controllers, respectively, which is due to the combination of controller type (activating/inhibiting) and controller configuration (inflow/outflow).

The responses in Figure 3 clusters into two groups, where the first group is the $E$-activating inflow controllers $1 / 3$ (black and red curves in Figures $3 \mathbf{a}$ and $3 \mathbf{b}$ ) and the $E$ inhibiting outflow controllers $6 / 8$ (blue and green curves in Figures $3 \mathbf{c}$ and $3 \mathbf{d}$ ). The second group is the $E$-inhibiting inflow controllers 2/4 (blue and green curves in Figures $3 \mathbf{a}$ and $3 \mathbf{b}$ ) and the $E$-activating outflow controllers $5 / 7$ (black and red curves in Figures $3 \mathbf{c}$ and $3 \mathbf{d}$ ). The reason why equally tuned controllers behaves slightly different is due to the nonlinearity of each individual controller combined with a relative large set point step change.

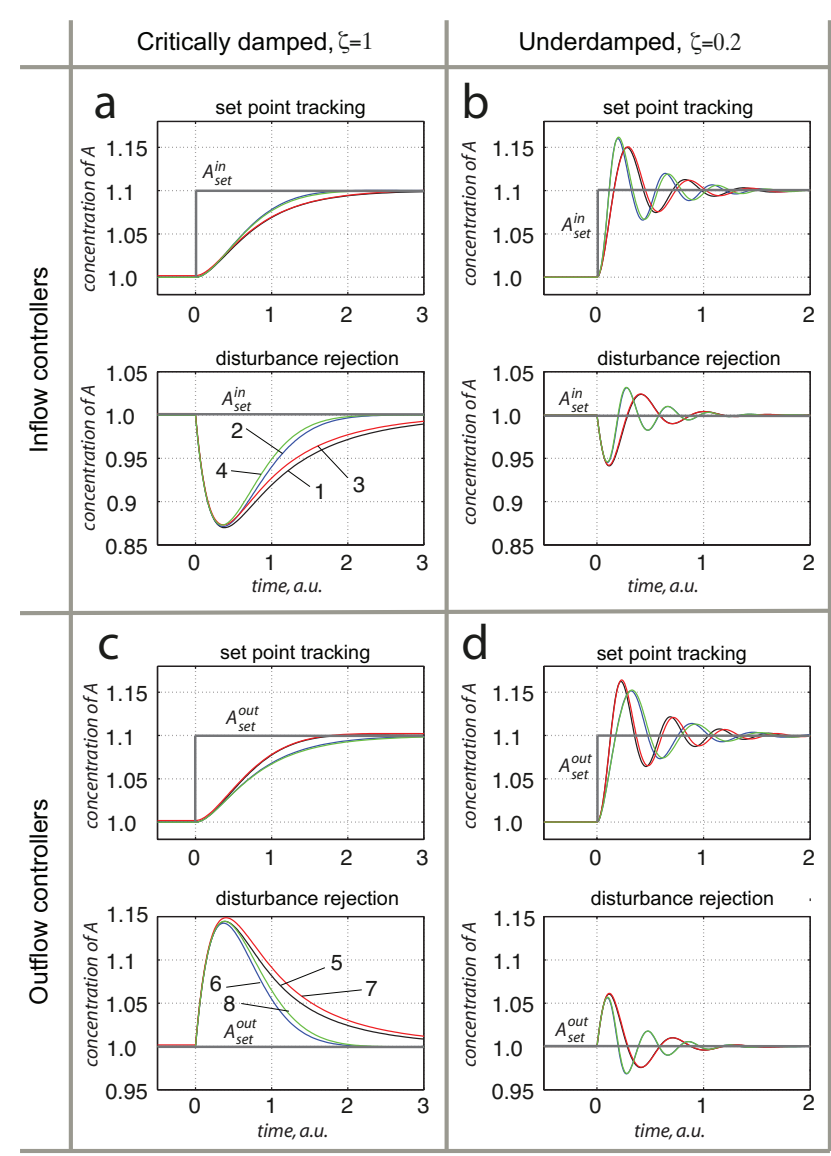

Figure 3. Dynamic properties of inflow and outflow controllers showing the response in concentration of species $A$. The color codes for the different inflow controller are: 1=black, $2=$ blue, $3=$ red and $4=$ green, and the color codes for the different outflow controllers are: $5=$ black, $6=$ blue, $7=$ red and $8=$ green. For the set point tracking curves, the set point changes from $A_{\text {set }}=1.0$ to $A_{\text {set }}=1.1$ at $t=0$. For the disturbance rejection curves, the disturbance is a unit step change from 5 to 6 at $t=0$ in $k_{\text {pert }}^{\text {out }}$ for inflow controllers and in $k_{\text {pert }}^{\text {in }}$ for outflow controllers. Panels a and b: Set point tracking (upper) and disturbance rejection (lower) responses for inflow controllers tuned for critically damped $(\zeta=1)$ and underdamped $(\zeta=0.2)$ responses, using the parameters shown in Table 2. Panels $\mathbf{c}$ and $\mathbf{d}$ : Set point tracking (upper) and disturbance rejection (lower) responses for outflow controllers tuned for critically damped $(\zeta=1)$ and underdamped $(\zeta=0.2)$ responses, using the parameters shown in Table 2.

From Table 1 we see that the inflow and outflow perturbations come into the expressions of $\omega_{n}$ and $\zeta$ in different ways. To visualize the effect of varying level of perturbation, Figure 4 shows dynamic responses of inflow controller 3 for $k_{\text {pert }}^{\text {out }}=\{3,5,7\}$ (Figure 4a) and outflow controller 6 for $k_{\text {pert }}^{\text {in }}=\{5,7,9\}$ (Figure $4 \mathbf{b}$ ). The effect of increased $k_{\text {pert }}^{\text {out }}$ for inflow controller 3 is slower dynamics with less damped response. On the other hand, outflow controller 6 shows faster dynamics together with more underdamped response at increased $k_{\text {pert }}^{\text {in }}$ levels. 


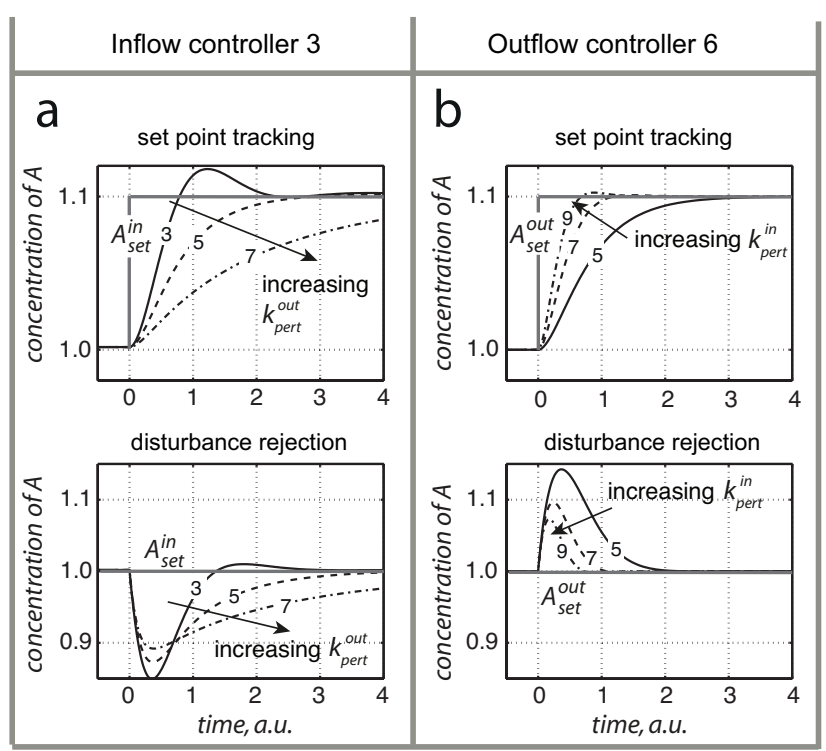

Figure 4. Set point tracking (upper) and disturbance rejection dynamics (lower) of species $A$ using inflow controller 3 (panel a) and outflow controller 6 (panel b) at different level of outflow and inflow perturbations, respectively. The set point change is a step from $A_{\text {set }}=1.0$ to $A_{\text {set }}=1.1$ at $t=0$ and the disturbance is a step increase of 1 from original value at $t=0$. In panel a the labeling on the curves corresponds to outflow perturbations of $k_{\text {pert }}^{\text {out }} \in\{3,5,7\}$. In panel $\mathbf{b}$ the labeling on the curves corresponds to inflow perturbation of $k_{\text {pert }}^{\text {in }} \in\{5,7,9\}$.

\section{Conclusions}

We have shown how a set of homeostatic controller motifs can be tuned, in a similar way as in industrial control systems, to exhibit a specified dynamic response with respect to overshoot $\delta$ and response time $T_{r}$. We have also shown analytically and through simulations how $i$ ) the level of inflow/outflow disturbances and $i i$ ) the values of different rate constants influence on the set point tracking properties. The corresponding disturbance rejection properties is also studied through simulations using a unit step input signal in the disturbance.

An important implication of the fact that all controller motifs can show identical dynamic responses is that one cannot postulate a specific controller motif based on measurement of disturbance rejection and/or set point tracking alone. The motif type, i.e. inflow or outflow, activating or inhibiting, rest on how the molecular mechanisms behind the controller interact and not on the system's ability to show a specific response. The specific response of physiological regulatory system is a result of tuning the system's kinetic parameters and the strength of the perturbation.

There is a great effort going on in both academia and industry to genetically manipulate organisms to produce useful bioproducts. One of the landmark studies published in Science last year was the implementation of the complete biosynthesis of opioids in yeast (Galanie et al., 2015; Service, 2015). Opioids like morphine are the primary drugs used for treatment of severe pain and pain manage- ment, and production depends on the cultivation of opium poppies. While the implementation of opioid biosynthesis in yeast is a tremendous achievement, it still requires an improvement in overall yield by a factor of $7 \cdot 10^{6}$ to compete with poppies (Galanie et al., 2015). Great improvements are expected (Galanie et al., 2015), but this will require an intricate tuning of the different parts of the biosynthesis pathway.

From a synthetic biology point of view, the work in this paper creates a basis one can use to identify which and how properties of a reaction and participating proteins/enzymes contributes to the dynamical response. For instance, the natural undamped frequency $\omega_{n}$, which is important for the swiftness of a controller motif, will for outflow controller 5 increase if we by some means manage to increase the production of $E$ (increase $k_{s}^{E}$ ) by e.g. increasing the expression of mRNA coding for $E$ (as shown in Table 1, a change in $k_{s}^{E}$ will also change the set point). A related example of such is reported in (Ang et al., 2010), where a two promotor network system is constructed in silico from realizable parts within the bacterium Escherichia coli. The network includes both basal rates and activated/repressed regulatory inputs, and hence, the network share similarities with inflow controller 2 in Figure 2. Two requirements were used as tuning criteria for the network, i.e. $\zeta=1$ (critically damped) and large $\omega_{n}$ indicating a response time $T_{r}$ as short as possible. In order to obtain the necessary approximate zero order degradation of the repressor $R$ (corresponding to our species $E$ ), two effectors $I_{1}$ and $I_{2}$ are included in order to force the repressor to work at saturated conditions, i.e. corresponding to the theoretical conditions, $K_{M}^{E_{s e t}}=0$, used in this paper.

An alternative approach to tuning is given in (Ang et al., 2013), where the tuning is related to the so-called response curves. These are steady state relationships between an input and an output variable, e.g. the molecular concentration of a transcription factor protein and the expressed protein, respectively, and not time dependent tuning as discussed in this paper. However, variations in kinetic parameter values results in different steady state relationships.

\section{References}

E. Ackerman, J.W. Rosevear, and W.F. McGuckin. A Mathematical Model of the Glucose-tolerance test. Physics in Medicine and Biology, 9(2):203-213, 1964.

I. Ajmera, M. Swat, C. Laibe, N. Le Novère, and V. Chelliah. The impact of mathematical modeling on the understanding of diabetes and related complications. CPT: Pharmacometrics \& Systems Pharmacology, 2 (7):14, 2013.

Standards of Medical Care in Diabetes-2014. American Diabetes Association, 2014. Diabetes Care 37:S14S80.

J. Ang and D.R. McMillen. Physical Constraints on Biological Integral Control Design for Homeostasis and 
Sensory Adaptation. Biophysical Journal, 104(2):505$515,2013$.

J. Ang, S. Bagh, B.P. Ingalls, and D.R. McMillen. Considerations for using integral feedback control to construct a perfectly adapting synthetic gene network. Journal of Theoretical Biology, 266(4):723-738, 2010.

J. Ang, E. Harris, B.J. Hussey, R. Kil, and D.R. McMillen. Tuning Response Curves for Synthetic Biology. ACS Synthetic Biology, 2(10):547-567, 2013.

E. Briese. Normal body temperature of rats: the setpoint controversy. Neuroscience \& Biobehavioral Reviews, 22(3):427-436, 1998.

W.B. Cannon. Organization for physiological homeostasis. Physiological reviews, IX:399-431, 1929.

T. Drengstig, I.W. Jolma, X.Y. Ni, K. Thorsen, X.M. Xu, and P. Ruoff. A Basic Set of Homeostatic Controller Motifs. Biophysical Journal, 103:2000-2010, 2012.

T. Drengstig, H.R. Ueda, and P. Ruoff. Predicting Perfect Adaptation Motifs in Reaction Kinetic Networks. Journal of Physical Chemistry B, 112(51):16752-16758, 2008.

S. Galanie, K. Thodey, I.J. Trenchard, M.F. Interrante, and C.D. Smolke. Complete biosynthesis of opioids in yeast. Science, 349(6252):1095-1100, 2015.

A.C. Guyton and J.E. Hall. Textbook of Medical Physiology. Elsevier Saunders, Philadelphia, PA, USA, 11 edition, 2006.

L.L. Langley. Homeostasis: Origins of the concept. John Wiley \& Sons, 1973.

M.O. Larsen, B. Rolin, M. Wilken, R.D. Carr, and C.F. Gotfredsen. Measurements of insulin secretory capacity and glucose tolerance to predict pancreatic $\beta$-cell mass in vivo in the nicotinamide/streptozotocin Göttingen minipig, a model of moderate insulin deficiency and diabetes. Diabetes, 52:118-123, 2003.

M. Mazumder and D.R. McMillen. Design and characterization of a dual-mode promoter with activation and repression capability for tuning gene expression in yeast. Nucleic Acids Research, 42(14):9514-9522, 2014.

R.F. Service. Modified yeast produce opiates from sugar. Science, 349(6249):677-677, 2015.

A. St Clair Gibson, J.H. Goedecke, Y.X. Harley, L.J. Myers, M.I. Lambert, T.D. Noakes, and E.V. Lambert. Metabolic setpoint control mechanisms in different physiological systems at rest and during exercise. Journal of Theoretical Biology, 236:60-72, 2005.
R. Steele. Influences of glucose loading and of injected insulin on hepatic glucose output. Annals of the New York Academy of Sciences, 82:420-430, 1959.

D.J. Thiele and D.H. Hamer. Tandemly duplicated upstream control sequences mediate copper-induced transcription of the Saccharomyces cerevisiae coppermetallothionein gene. Molecular and Cellular Biology, 6(4):1158-1163, 1986.

K. Thorsen. Controller Motifs for Homeostatic Regulation and Their Applications in Biological Systems. PhD thesis, University of Stavanger, 2015.

K. Thorsen, P. Ruoff, and T. Drengstig. Control theoretic properties of physiological controller motifs. In 2013 International Conference on System Science and Engineering (ICSSE), pages 165-170. IEEE, 2013.

B.G. Topp, M.D. McArthur, and D.T. Finegood. Metabolic adaptations to chronic glucose infusion in rats. Diabetologia, 47(9):1602-1610, 2004. 